\title{
On the Field-theoreticity of Homomorphisms between the Multiplicative Groups of Number Fields
}

\author{
by
}

Yuichiro HoshI

\begin{abstract}
We discuss the field-theoreticity of homomorphisms between the multiplicative groups of number fields. We prove that, for instance, for a given isomorphism between the multiplicative groups of number fields, either the isomorphism or its multiplicative inverse arises from an isomorphism of fields if and only if the given isomorphism is SPU-preserving (i.e., roughly speaking, preserves the subgroups of principal units with respect to various nonarchimedean primes).
\end{abstract}

2010 Mathematics Subject Classification: Primary 11R04.

Keywords: number field, multiplicative group, field-theoreticity, PU-preserving homomorphism.

\section{Introduction}

In the present paper, we discuss the field-theoreticity of homomorphisms between the multiplicative groups of fields. Let us consider the following problem:

For a homomorphism $\alpha$ : ${ }^{\circ} k^{\times} \rightarrow{ }^{\bullet} k^{\times}$between the multiplicative groups of fields ${ }^{\circ} k$ and ${ }^{\bullet} k$, when does $\alpha$ arise from a homomorphism of fields ${ }^{\circ} k \rightarrow{ }^{\bullet} k$ ? In other words, when is the additive structure of ${ }^{\circ} k$ compatible with the additive structure of $\bullet k$ relative to $\alpha$ ?

At a more philosophical level:

How can one understand the additive structure of a field in the language of the multiplicative structure of the field?

Communicated by S. Mochizuki. Received January 23, 2013. Revised July 23, 2013.

Y. Hoshi: Research Institute for Mathematical Sciences, Kyoto University, Kyoto 606-8502, Japan;

e-mail: yuichiro@kurims.kyoto-u.ac.jp

(C) 2014 Research Institute for Mathematical Sciences, Kyoto University. All rights reserved. 
Now let us recall the following consequence of Uchida's lemma (reviewed in [1, Proposition 1.3]) that is implicit in the argument of [7, Lemmas 8-11] (cf. also [5, Lemma 4.7]):

For $\square \in\{\circ, \bullet\}$, let ${ }^{\square} k$ be an algebraically closed field and ${ }^{\square} C$ a projective smooth curve over ${ }^{\square} k$. Write ${ }^{\square} K$ for the function field of ${ }^{\square} C$, and ${ }^{\square} C^{\text {cl }}$ for the set of closed points of ${ }^{\square} C$. For each ${ }^{\square} x \in{ }^{\square} C^{\text {cl }}$, write $\mathcal{O}_{\square}{ }_{C,}{ }_{x} \subseteq{ }^{\square} K$ for the local ring of ${ }^{\square} C$ at ${ }^{\square} x, \mathfrak{m}_{\square}{ }_{C,{ }_{x}} \subseteq \mathcal{O}_{\square_{C,},{ }_{x}}$ for the maximal ideal of $\mathcal{O}_{\square} C_{\square_{x}}$, and ord $_{{ }_{x}}:{ }^{\square} K^{\times} \rightarrow \mathbb{Z}$ for the valuation of ${ }^{\square} K$ given by mapping $f \in{ }^{\square} K^{\times}$to the order of $f$ at ${ }^{\square} x \in{ }^{\square} C$. (Thus, one verifies easily that $1+\mathfrak{m}_{\square} \square_{,{ }_{x}} \subseteq \operatorname{Ker}\left(\operatorname{ord}_{\square_{x}}\right)=\mathcal{O}_{\square}^{\times}{ }_{C, \square x} \subseteq{ }^{\square} K^{\times}$.) Let

$$
\alpha:{ }^{\circ} K^{\times} \stackrel{\sim}{\rightarrow} \cdot K^{\times}
$$

be an isomorphism between the multiplicative groups of ${ }^{\circ} K,{ }^{\bullet} K$. Then $\alpha$ arises from an isomorphism of fields ${ }^{\circ} K \stackrel{\sim}{\rightarrow} \cdot K$ if and only if there exists a bijection $\phi:{ }^{\bullet} C^{\mathrm{cl}} \stackrel{\sim}{\longrightarrow}{ }^{\circ} C^{\text {cl }}$ such that, for all ${ }^{\bullet} x \in{ }^{\bullet} C^{\text {cl }}$ and ${ }^{\circ} x:=$ $\phi\left(\bullet^{\bullet} x\right) \in{ }^{\circ} C^{\text {cl }}$, we have ord $\circ_{x}=\operatorname{ord} \bullet_{x} \circ \alpha$ and $1+\mathfrak{m}_{\circ} C,{ }^{\circ} x=\alpha^{-1}\left(1+\mathfrak{m} \bullet C, \bullet_{x}\right)$.

Moreover, the issue of recovering the additive structure not only for isomorphisms (as in the above result) but also for suitable homomorphisms between the multiplicative groups of function fields has been intensively studied by M. Saïdi and A. Tamagawa in, for instance, $[3, \S 4]$ and $[4, \S 5]$ (cf. Remark 3.3.1).

In the present paper, we discuss an analogue for number fields of the above result. In the remainder of this paper, let Primes be the set of all prime numbers, $\square \in\{\circ, \bullet\},{ }^{\square} k$ a number field (i.e., a finite extension of $\mathbb{Q}$ ), $\square_{\mathfrak{o}} \subseteq{ }^{\square} k$ the ring of integers of $\square k, \square \mathcal{V}$ the set of maximal ideals of $\square_{\mathfrak{o}}$ (i.e., the set of nonarchimedean primes of $\square k$ ), and $\square \mathbb{Q} \subseteq \square k$ the (uniquely determined) subfield of ${ }^{\square} k$ that is isomorphic to $\mathbb{Q}$. For $\square_{\mathfrak{p}} \in{ }^{\square} \mathcal{V}$, write ${ }^{\square} \mathfrak{o}_{\square_{\mathfrak{p}}}$ for the localization of $\square_{\mathfrak{o}}$ at ${ }^{\square} \mathfrak{p}$, $\mathfrak{c}\left({ }^{\square}\right)$ for the residue characteristic of $\square_{\mathfrak{p}}$ (thus, we have a map $\mathfrak{c}:{ }^{\square} \mathcal{V} \rightarrow$ Primes), and $\operatorname{ord}_{\square \mathfrak{p}}:{ }^{\circ} k^{\times} \rightarrow \mathbb{Z}$ for the (uniquely determined) surjective valuation of $\square k$ associated to $\square_{\mathfrak{p}}$ (cf. Definition 1.1). Let

$$
\alpha:{ }^{\circ} k^{\times} \rightarrow{ }^{\bullet} k^{\times}
$$

be a homomorphism of multiplicative groups.

Theorem A. The following conditions are equivalent:

(1) The homomorphism $\alpha$ arises from a homomorphism of fields ${ }^{\circ} k \rightarrow{ }^{\bullet} k$.

(2) The homomorphism $\alpha$ is CPU-preserving (i.e., there is a map $\phi:{ }^{\bullet} \mathcal{V} \rightarrow{ }^{\circ} \mathcal{V}$ such that $\mathfrak{c}(\bullet \mathfrak{p})=\mathfrak{c}(\phi(\bullet \mathfrak{p}))$ for every $\bullet \mathfrak{p} \in \bullet \mathcal{V}$, and moreover the inclusion 
$1+{ }^{\circ} \mathfrak{p}^{\circ} \mathfrak{o}_{\mathfrak{p}} \subseteq \alpha^{-1}\left(1+\bullet^{\bullet} \bullet^{\bullet} \bullet_{\mathfrak{p}}\right)$, where ${ }^{\circ} \mathfrak{p}:=\phi(\bullet \mathfrak{p}) \in{ }^{\circ} \mathcal{V}$, holds for all but finitely many $\mathfrak{\bullet} \in \bullet \mathcal{V}$ - cf. Definition 1.3(ii)), and there exists an $x \in \mathbb{Q}^{\times} \backslash \mathbb{Z}^{\times}$such that the " $x$ " in ${ }^{\circ} k$ maps, via $\alpha$, to the " $x$ " in $* k$.

(3) The homomorphism $\alpha$ is PU-preserving (i.e., there exists a map $\phi:{ }^{\bullet} \mathcal{V} \rightarrow{ }^{\circ} \mathcal{V}$ such that the inclusion $1+{ }^{\circ} \mathfrak{p}^{\circ} \mathfrak{o}_{\mathfrak{p}} \subseteq \alpha^{-1}\left(1+\bullet^{\bullet} \bullet^{\bullet} \bullet_{\mathfrak{p}}\right)$, where ${ }^{\circ} \mathfrak{p}:=\phi\left(\bullet_{\mathfrak{p}}\right) \in$ ${ }^{\circ} \mathcal{V}$, holds for all but finitely many $\mathfrak{p}_{\mathfrak{p}} \in \boldsymbol{\bullet}^{\bullet} \mathcal{V}$ - cf. Definition 1.3(i)), and the restriction ${ }^{\circ} \mathbb{Q}^{\times} \rightarrow{ }^{\bullet} k^{\times}$of $\alpha$ to ${ }^{\circ} \mathbb{Q}^{\times} \subseteq{ }^{\circ} k^{\times}$arises from a homomorphism of fields ${ }^{\circ} \mathbb{Q} \rightarrow \bullet k$.

By concentrating on surjections, we obtain the following result (cf. Corollary 3.2$)$.

Theorem B. Suppose that the homomorphism $\alpha$ is surjective. Then either $\alpha$ or the composite $(-)^{-1} \circ \alpha$ (i.e., the surjection ${ }^{\circ} k^{\times} \rightarrow{ }^{\bullet} k^{\times}$obtained by mapping $x \in{ }^{\circ} k^{\times}$to $\alpha(x)^{-1} \in{ }^{\bullet} k^{\times}$) arises from an isomorphism of fields ${ }^{\circ} k \stackrel{\sim}{\rightarrow} \bullet$ if and only if the surjection $\alpha$ is SPU-preserving (i.e., there exists a map $\phi:{ }^{\bullet} \mathcal{V} \rightarrow{ }^{\circ} \mathcal{V}$ such that the equality $1+{ }^{\circ} \mathfrak{p}^{\circ} \mathfrak{o}_{{ }^{o}}=\alpha^{-1}\left(1+{ }^{\bullet} \mathfrak{p}{ }^{\bullet} \bullet_{\mathfrak{p}}\right)$, where ${ }^{\circ} \mathfrak{p}:=\phi(\bullet \mathfrak{p}) \in{ }^{\circ} \mathcal{V}$, holds for all but finitely many $\bullet_{\mathfrak{p}} \in \boldsymbol{\bullet}^{\mathcal{V}}-$ cf. Definition $\left.1.3(\mathrm{i})\right)$.

As corollaries of Theorem A, we also prove the following results, that may be regarded as analogues of Uchida's lemma for number fields (cf. Theorem 3.1 and Corollary 3.3).

Theorem C. The homomorphism $\alpha$ arises from a homomorphism of fields ${ }^{\circ} k \rightarrow{ }^{\bullet} k$ if and only if there exists a map $\phi:{ }^{\bullet} \mathcal{V} \rightarrow{ }^{\circ} \mathcal{V}$ over $\mathfrak{P r i m e s}$ relative to $\mathfrak{c}$ (i.e., $\mathfrak{c}\left(\bullet^{\mathfrak{p}}\right)=\mathfrak{c}(\phi(\bullet \mathfrak{p}))$ for every $\left.\bullet_{\mathfrak{p}} \in{ }^{\bullet} \mathcal{V}\right)$ such that, for $\bullet_{\mathfrak{p}} \in{ }^{\bullet} \mathcal{V}$, if ${ }^{\circ} \mathfrak{p}:=\phi\left({ }^{\bullet} \mathfrak{p}\right) \in{ }^{\circ} \mathcal{V}$, then

$$
\operatorname{ord}_{{ }_{\mathfrak{p}}}=\operatorname{ord}_{\bullet \mathfrak{p}} \circ \alpha
$$

for infinitely many $\bullet_{\mathfrak{p}} \in \boldsymbol{\bullet} \mathcal{V}$, and

$$
1+{ }^{\circ} \mathfrak{p}^{\circ} \mathfrak{o}_{\mathfrak{p}} \subseteq \alpha^{-1}\left(1+\mathfrak{p}^{\bullet} \mathfrak{o}_{\mathfrak{p}}\right)
$$

for all but finitely many $\bullet \mathfrak{p} \in \bullet \mathcal{V}$.

Theorem D. Suppose that the homomorphism $\alpha$ is surjective. Then it arises from an isomorphism of fields ${ }^{\circ} k \stackrel{\sim}{\rightarrow} \cdot k$ if and only if there exists $\phi:{ }^{\bullet} \mathcal{V} \rightarrow{ }^{\circ} \mathcal{V}$ such that, for $\bullet \mathfrak{p} \in \bullet^{\bullet} \mathcal{V}$, if ${ }^{\circ} \mathfrak{p}:=\phi(\bullet \mathfrak{p}) \in{ }^{\circ} \mathcal{V}$, then

$$
1+{ }^{\circ} \mathfrak{p}^{\circ} \mathfrak{o}_{\mathfrak{p}}=\alpha^{-1}\left(1+\mathfrak{p}^{\bullet} \mathfrak{o} \cdot \mathfrak{p}\right)
$$

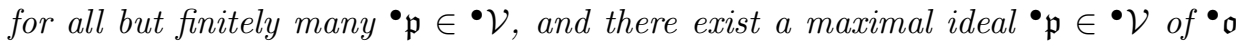
and a positive integer $n$ such that

$$
n \cdot \operatorname{ord}_{\circ}=\operatorname{ord}_{\bullet p} \circ \alpha .
$$




\section{§1. PU-preserving homomorphisms}

In this section, we define and discuss the notion of a $P U$-preserving homomorphism (cf. Definition 1.3(i) below). Throughout, we use the notation specified in the Introduction before the statement of Theorem A. Moreover, let $k$ be a number field; we shall use similar notation $\mathfrak{o} \subseteq k, \mathcal{V}$ for objects associated to $k$.

Definition 1.1. Let $\mathfrak{p} \in \mathcal{V}$ be a maximal ideal of $\mathfrak{o}$.

(i) We shall write $\mathfrak{o}_{\mathfrak{p}}$ for the localization of $\mathfrak{o}$ at $\mathfrak{p}$,

$$
\kappa(\mathfrak{p}):=\mathfrak{o} / \mathfrak{p} \stackrel{\sim}{\longrightarrow} \mathfrak{o}_{\mathfrak{p}} / \mathfrak{p o}_{\mathfrak{p}}
$$

for the residue field of $\mathfrak{o}$ at $\mathfrak{p}$, and

$$
\mathfrak{c}(\mathfrak{p}):=\operatorname{char}(\kappa(\mathfrak{p}))
$$

for the characteristic of $\kappa(\mathfrak{p})$. Thus, we have a map

$$
\mathfrak{c}: \mathcal{V} \rightarrow \mathfrak{P r i m e s}
$$

(ii) We shall write

$$
\operatorname{ord}_{\mathfrak{p}}: k^{\times} \rightarrow \mathbb{Z}
$$

for the (uniquely determined) surjective valuation of $k$ associated to $\mathfrak{p}$. Thus, one verifies easily that the kernel $\operatorname{Ker}\left(\operatorname{ord}_{\mathfrak{p}}\right) \subseteq k^{\times}$coincides with the group $\mathfrak{o}_{\mathfrak{p}}^{\times} \subseteq k^{\times}$ of invertible elements of $\mathfrak{o}_{\mathfrak{p}}$ (cf. (i)), i.e.,

$$
\operatorname{Ker}\left(\operatorname{ord}_{\mathfrak{p}}\right)=\mathfrak{o}_{\mathfrak{p}}^{\times} \subseteq k^{\times} .
$$

Moreover, we have a natural exact sequence of abelian groups

$$
1 \rightarrow 1+\mathfrak{p o}_{\mathfrak{p}} \rightarrow \operatorname{Ker}\left(\operatorname{ord}_{\mathfrak{p}}\right) \rightarrow \kappa(\mathfrak{p})^{\times} \rightarrow 1 .
$$

Remark 1.1.1. By the map $\mathfrak{c}$ (cf. Definition 1.1(i)), let us identify $\mathfrak{P r i m e s}$ with the "V" that occurs in the case where we take the " $k$ " to be a number field that is isomorphic to the field of rational numbers (e.g., ${ }^{\square} \mathbb{Q}$ ).

Definition 1.2. Let $\phi:{ }^{\bullet} \mathcal{V} \rightarrow{ }^{\circ} \mathcal{V}$ be a map of sets. Then we shall say that $\phi$ is characteristic-compatible if $\phi$ is a map over $\mathfrak{P r i m e s}$ (relative to $\mathfrak{c}$ - cf. Definition 1.1(i)), i.e., the diagram

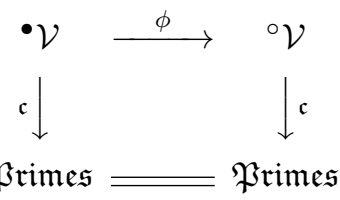

commutes. 
Remark 1.2.1. One verifies easily that if $\phi: \bullet \mathcal{V} \rightarrow{ }^{\circ} \mathcal{V}$ is characteristic-compatible then it is finite-to-one, i.e., the inverse image of any element of ${ }^{\circ} \mathcal{V}$ is finite.

Definition 1.3. Let $\alpha:{ }^{\circ} k^{\times} \rightarrow{ }^{\bullet} k^{\times}$be a homomorphism of groups.

(i) Let $\phi: \bullet \mathcal{V} \rightarrow{ }^{\circ} \mathcal{V}$ be a map of sets. Then we shall say that the homomorphism $\alpha$ is $(\phi-) P U$-preserving ("principal-unit-preserving") [respectively, $(\phi-) S P U$ preserving ("strictly principal-unit-preserving")] if the inclusion $1+{ }^{\circ} \mathfrak{p}^{\circ} \mathfrak{o}_{\circ} \mathfrak{p} \subseteq$ $\alpha^{-1}\left(1+\mathfrak{\bullet}^{\bullet} \mathfrak{o}_{\mathfrak{p}}\right)\left[\right.$ respectively, the equality $\left.1+{ }^{\circ} \mathfrak{p}^{\circ} \mathfrak{o}_{\mathfrak{o}_{\mathfrak{p}}}=\alpha^{-1}\left(1+\bullet_{\mathfrak{p}} \mathfrak{o}_{\mathfrak{p}}\right)\right]$, where ${ }^{\circ} \mathfrak{p}:=\phi\left(\bullet_{\mathfrak{p}}\right) \in{ }^{\circ} \mathcal{V}$, holds for all but finitely many $\bullet_{\mathfrak{p}} \in \bullet^{\bullet} \mathcal{V}$. If, in this situation, for some $\bullet_{\mathfrak{p}} \in \bullet^{\bullet} \mathcal{V}$, the inclusion $1+{ }^{\circ} \mathfrak{p}^{\circ} \mathfrak{o}_{\mathfrak{p}} \subseteq \alpha^{-1}\left(1+\bullet_{\mathfrak{p}} \mathfrak{o} \mathfrak{p}\right)$ [respectively, the equality $\left.1+{ }^{\circ} \mathfrak{p}^{\circ} \mathfrak{o}_{\circ \mathfrak{p}}=\alpha^{-1}\left(1+\mathfrak{p}^{\bullet} \mathfrak{o}_{\mathfrak{p}}\right)\right]$ does not hold, then we shall say that $\bullet_{\mathfrak{p}}$ is $P U$-exceptional [respectively, $S P U$-exceptional] for $(\alpha, \phi)$.

(ii) We shall say that the homomorphism $\alpha$ is CPU-preserving ("characteristic-compatibly principal-unit-preserving") if $\alpha$ is $\phi$-PU-preserving for some characteristic-compatible map $\phi: \bullet \mathcal{V} \rightarrow{ }^{\circ} \mathcal{V}$.

Remark 1.3.1. In the notation of Definition 1.3, one verifies easily that if $\alpha$ is $\phi$-PU-preserving, and $\mathfrak{c}(\bullet \mathfrak{p})=\mathfrak{c}(\phi(\bullet \mathfrak{p}))$ for all but finitely many $\bullet \mathfrak{p} \in \bullet \mathcal{V}$, thenafter replacing $\phi$ by a suitable extension (to a map ${ }^{\bullet} \mathcal{V} \rightarrow{ }^{\circ} \mathcal{V}$ ) of the restriction of $\phi$ to the subset of $\bullet \mathcal{V}$ consisting of $\bullet \mathfrak{p} \in \bullet \mathcal{V}$ such that $\mathfrak{c}(\bullet \mathfrak{p})=\mathfrak{c}(\phi(\bullet \mathfrak{p}))$ - $\alpha$ is CPU-preserving.

Lemma 1.4. Let $\iota:{ }^{\circ} k \rightarrow{ }^{\bullet} k$ be a homomorphism of fields. Write $\iota^{\times}:{ }^{\circ} k^{\times} \rightarrow$ $\bullet k^{\times}$for the homomorphism induced by $\iota$, and $\mathcal{V}_{\iota}: \bullet \mathcal{V} \rightarrow{ }^{\circ} \mathcal{V}$ for the (necessarily surjective and characteristic-compatible) map obtained by mapping $\bullet \mathfrak{p} \in \bullet \mathcal{V}$ to $\iota^{-1}(\bullet \mathfrak{p}) \cap{ }^{\circ} \mathfrak{o} \in{ }^{\circ} \mathcal{V}$. Then, for every ${ }^{\bullet} \mathfrak{p} \in{ }^{\bullet} \mathcal{V}$,

$$
1+\mathcal{V}_{\iota}(\bullet \mathfrak{p})^{\circ} \mathfrak{o}_{\mathcal{V}}(\bullet \mathfrak{p})=\left(\iota^{\times}\right)^{-1}\left(1+\bullet_{\mathfrak{p}} \bullet \mathfrak{o}_{\mathfrak{p}}\right) .
$$

In particular, $\iota^{\times}$is $\mathcal{V}_{\iota}$-SPU-preserving and CPU-preserving.

Proof. This follows immediately from the various definitions involved.

Lemma 1.5. Let $\alpha:{ }^{\circ} k^{\times} \rightarrow{ }^{\bullet} k^{\times}$be a homomorphism of groups, $\phi:{ }^{\bullet} \mathcal{V} \rightarrow{ }^{\circ} \mathcal{V}$ a map of sets, and $\bullet \mathfrak{p} \in \bullet^{\bullet} \mathcal{V}$. Write ${ }^{\circ} \mathfrak{p}:=\phi(\bullet \mathfrak{p}) \in{ }^{\circ} \mathcal{V}$.

(i) Suppose that $\alpha$ is $\boldsymbol{\phi}$-PU-preserving, and $\bullet \mathfrak{p}$ is not PU-exceptional for $(\alpha, \phi)$. Then $\operatorname{Ker}\left(\operatorname{ord}_{\iota_{\mathfrak{p}}}\right) \subseteq \alpha^{-1}\left(\operatorname{Ker}\left(\operatorname{ord} \bullet_{\mathfrak{p}}\right)\right)$. In particular, $\alpha$ determines homomorphisms of groups

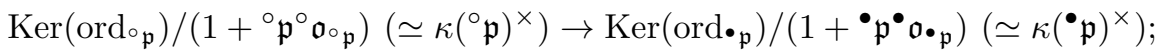

$$
\begin{aligned}
& { }^{\circ} k^{\times} / \operatorname{Ker}\left(\operatorname{ord} \circ_{\mathfrak{p}}\right)(\simeq \mathbb{Z}) \rightarrow \bullet k^{\times} / \operatorname{Ker}\left(\operatorname{ord} \bullet_{\mathfrak{p}}\right)(\simeq \mathbb{Z}) .
\end{aligned}
$$


(ii) Suppose that $\alpha$ is $\boldsymbol{\phi}$-SPU-preserving, and $\bullet \mathfrak{p}$ is not SPU-exceptional for $(\alpha, \phi)$. Suppose, moreover, that $\alpha$ is surjective. Then the two displayed homomorphisms of (i) are isomorphisms. Moreover, $\alpha$ is CPU-preserving.

Proof. Assertion (i) follows immediately from the (easily verified) fact that, for each $\square \in\{\circ, \bullet\}$, the subgroup $\operatorname{Ker}\left(\operatorname{ord} \square_{\mathfrak{p}}\right) /\left(1+{ }^{\square} \mathfrak{p}^{\square} \mathfrak{o}_{\square \mathfrak{p}}\right) \subseteq{ }^{\square} k^{\times} /\left(1+{ }^{\square} \mathfrak{p}^{\square} \mathfrak{o}_{\square_{\mathfrak{p}}}\right)$ coincides with the maximal torsion subgroup of $\square k^{\times} /\left(1+{ }^{\square} \mathfrak{p}^{\square} \mathfrak{o}_{\square \mathfrak{p}}\right)$.

Next, we verify (ii). The assertion that the two displayed homomorphisms of (i) are isomorphisms follows immediately from the various definitions involved, together with the (easily verified) fact that every surjective endomorphism of $\mathbb{Z}$ is an isomorphism. The assertion that $\alpha$ is CPU-preserving follows immediately from Remark 1.3.1, together with the fact that if $\kappa\left({ }^{\circ} \mathfrak{p}\right)^{\times}$is isomorphic to $\kappa\left({ }^{\bullet} \mathfrak{p}\right)^{\times}$, then $\mathfrak{c}\left({ }^{\circ} \mathfrak{p}\right)=\mathfrak{c}\left({ }^{\bullet} \mathfrak{p}\right)$.

Lemma 1.6. Let $\phi:{ }^{\bullet} \mathcal{V} \rightarrow{ }^{\circ} \mathcal{V}$ be a map of sets and $\alpha, \beta:{ }^{\circ} k^{\times} \rightarrow{ }^{\bullet} k^{\times}$homomorphisms of groups. Suppose that $\alpha$ and $\beta$ are $\boldsymbol{\phi}$-PU-preserving. Then the homomorphism $\alpha \cdot \beta:{ }^{\circ} k^{\times} \rightarrow{ }^{\bullet} k^{\times}$(mapping $x \in{ }^{\circ} k^{\times}$to $\alpha(x) \cdot \beta(x) \in{ }^{\bullet} k^{\times}$) is $\phi$-PU-preserving.

Proof. This follows immediately from the various definitions involved.

Remark 1.6.1. In the situation of Lemma 1.6:

(i) In general, the product of two $\phi$-SPU-preserving homomorphisms is not $\phi$-SPU-preserving. Indeed, although the identity automorphism $\operatorname{id}_{\mathbb{Q}^{\times}}$of $\mathbb{Q}^{\times}$is $\mathrm{id}_{\mathfrak{P r i m e s}}-S P U$-preserving (cf. Remark 1.1.1), the product of two id $\mathbb{Q}_{\mathbb{Q}}$ 's (which maps $x \in \mathbb{Q}^{\times}$to $\left.x^{2} \in \mathbb{Q}^{\times}\right)$is not $\mathrm{id}_{\mathfrak{P r i m e s}}-S P U$-preserving.

(ii) Moreover, in general, the product of CPU-preserving homomorphisms is not $C P U$-preserving. Indeed, suppose that $k$ is Galois over $\mathbb{Q}$. Then it follows from Lemma 1.4 that the automorphism $g^{\times}$of $k^{\times}$determined by an element $g \in \operatorname{Gal}(k / \mathbb{Q})$ of $\operatorname{Gal}(k / \mathbb{Q})$ is $C P U$-preserving. Write Nm for the product of all such automorphisms $g^{\times}$. (Thus, Nm is the composite of the norm map $k^{\times} \rightarrow \mathbb{Q}^{\times}$ and the natural inclusion $\mathbb{Q}^{\times} \hookrightarrow k^{\times}$.) Assume that the difference $\delta: k^{\times} \rightarrow k^{\times}$ of $\mathrm{Nm}$ and the endomorphism of $k^{\times}$given by mapping $x \in k^{\times}$to $x^{[k: \mathbb{Q}]} \in k^{\times}$ is $C P U$-preserving. Then one verifies immediately that the restriction of $\delta$ to the subgroup $\mathbb{Q}^{\times} \subseteq k^{\times}$is trivial. Thus, it follows immediately from Proposition 2.4(i) below that we obtain a contradiction.

Definition 1.7. Let $\phi:{ }^{\bullet} \mathcal{V} \rightarrow{ }^{\circ} \mathcal{V}$ be a map of sets. Then we shall write

$$
\operatorname{Hom}\left({ }^{\circ} k^{\times}, \bullet k^{\times}\right)
$$


for the (abelian) group of all group homomorphisms ${ }^{\circ} k^{\times} \rightarrow{ }^{\bullet}$, and

$$
\operatorname{Hom}^{\phi-\mathrm{PU}}\left({ }^{\circ} k^{\times},{ }^{\bullet} k^{\times}\right) \subseteq \operatorname{Hom}\left({ }^{\circ} k^{\times}, \bullet k^{\times}\right)
$$

for the subgroup (cf. Lemma 1.6) of $\phi$-PU-preserving homomorphisms ${ }^{\circ} k^{\times} \rightarrow k^{\times}$.

Lemma 1.8. Let $\phi:^{\bullet} \mathcal{V} \rightarrow{ }^{\circ} \mathcal{V}$ be a map of sets. Then the homomorphism of groups

$$
\operatorname{Hom}^{\phi-\mathrm{PU}}\left({ }^{\circ} k^{\times},{ }^{\bullet} k^{\times}\right) \rightarrow \operatorname{Hom}\left({ }^{\circ} \mathbb{Q}^{\times},{ }^{\bullet} k^{\times}\right)
$$

induced by the natural inclusion ${ }^{\circ} \mathbb{Q}^{\times} \hookrightarrow{ }^{\circ} k^{\times}$factors through the subgroup $\operatorname{Hom}^{(\mathfrak{c o} \phi)-\mathrm{PU}}\left({ }^{\circ} \mathbb{Q}^{\times},{ }^{\bullet} k^{\times}\right) \subseteq \operatorname{Hom}\left({ }^{\circ} \mathbb{Q}^{\times},{ }^{\bullet} k^{\times}\right)(c f$. Remark 1.1.1). In particular, we obtain a homomorphism of groups

$$
\operatorname{Hom}^{\phi-\mathrm{PU}}\left({ }^{\circ} k^{\times}, \bullet k^{\times}\right) \rightarrow \operatorname{Hom}^{(\mathfrak{c} \circ \phi)-\mathrm{PU}}\left({ }^{\circ} \mathbb{Q}^{\times}, \bullet k^{\times}\right) .
$$

Proof. This follows immediately from the various definitions involved.

\section{§2. Field-theoreticity for certain PU-preserving homomorphisms}

In this section, we prove the field-theoreticity of certain PU-preserving homomorphisms (cf. Theorem 2.5 below). We maintain the notation of $\S 1$.

Lemma 2.1. Let $\phi:^{\bullet} \mathcal{V} \rightarrow{ }^{\circ} \mathcal{V}$ be a map of sets, $n$ a positive integer, and let $x_{1}, \ldots, x_{n} \in{ }^{\circ} k^{\times}$. Suppose that the image of the composite $\bullet \mathcal{V} \stackrel{\phi}{\rightarrow}{ }^{\circ} \mathcal{V} \stackrel{\mathfrak{c}}{\rightarrow}$ Primes is of density one. Then the subset $S\left[\phi ; x_{1}, \ldots, x_{n}\right] \subseteq \bullet \mathcal{V}$ consisting of all maximal ideals $\bullet_{\mathfrak{p}}$ of $\bullet^{\mathfrak{o}}$ that satisfy the following condition is infinite: If ${ }^{\circ} \mathfrak{p}:=\phi\left(\bullet^{\mathfrak{p}}\right) \in{ }^{\circ} \mathcal{V}$, then $x_{i} \in \operatorname{Ker}\left(\operatorname{ord}_{\circ}\right)$ for each $i \in\{1, \ldots, n\}$, and $\sharp \kappa\left({ }^{\circ} \mathfrak{p}\right)=\mathfrak{c}\left({ }^{\circ} \mathfrak{p}\right)$.

Proof. One verifies immediately that, to prove Lemma 2.1, it suffices to verify that the set of those $p \in \mathfrak{P r i m e s}$ that split completely in the finite extension ${ }^{\circ} \mathrm{k} /{ }^{\circ} \mathbb{Q}$ contains a subset of $\mathfrak{P r i m e s}$ of positive density. The latter fact follows immediately, by considering the Galois closure of ${ }^{\circ} k /{ }^{\circ} \mathbb{Q}$, from Chebotarev's density theorem.

Lemma 2.2. For $p \in \mathfrak{P r i m e s}$, write $\operatorname{ord}_{p}: \mathbb{Q}^{\times} \rightarrow \mathbb{Z}$ for the surjective $p$-adic valuation. Let $x, y \in \mathbb{Q}^{\times}$with $y \notin\{1,-1\}$. Then the subset $S_{x,\langle y\rangle} \subseteq$ Primes consisting of all $p \in \mathfrak{P r i m e s}$ that satisfy the following condition is infinite: $x, y \in$ $\operatorname{Ker}\left(\operatorname{ord}_{p}\right)$, and the image of $x$ in $\mathbb{F}_{p}^{\times}$is contained in the subgroup of $\mathbb{F}_{p}^{\times}$generated by the image of $y$ in $\mathbb{F}_{p}^{\times}$.

Proof. This follows from the proof of [2, Theorem 1]. For the reader's convenience (and to make it clear that the argument given in [2] works under our assumption $y \notin\{1,-1\})$, we review the argument as follows: 
Since $y \notin\{1,-1\}$, it is immediate that, by replacing $y$ by $y^{-1}$ if necessary, we may assume that $|y|>1$. Write $\left(x_{1}, x_{2}\right),\left(y_{1}, y_{2}\right)$ for the (uniquely determined) pairs of nonzero rational integers such that $x_{1} \mathbb{Z}+x_{2} \mathbb{Z}=\mathbb{Z}, y_{1} \mathbb{Z}+y_{2} \mathbb{Z}=\mathbb{Z}$, $x_{2}, y_{2}>0, x=x_{1} / x_{2}, y=y_{1} / y_{2}$. For each nonnegative integer $n$, write $a_{n}:=$ $x_{1} \cdot y_{2}^{n}-x_{2} \cdot y_{1}^{n}$. Now if $a_{n}=0$ for some $n$, then Lemma 2.2 is immediate. Thus, assume that $a_{n} \neq 0$ for every $n$. Next, observe that $S_{x,\langle y\rangle}$ coincides with the set of $p \in \mathfrak{P r i m e s}$ such that $x, y \in \operatorname{Ker}\left(\operatorname{ord}_{p}\right)$ but $a_{n} \notin \operatorname{Ker}\left(\operatorname{ord}_{p}\right)$ for some $n$.

To verify Lemma 2.2, assume towards a contradiction that $S_{x,\langle y\rangle}$ is finite. Write $n_{0}:=\sharp\left(\mathbb{Z} /\left(\prod_{p \in S_{x,\langle y\rangle}} p^{\text {ord }_{p}\left(a_{0}\right)+1}\right) \mathbb{Z}\right)^{\times}$. (One verifies easily that, for every $p \in S_{x,\langle y\rangle}$ and $z \in \mathbb{Q}^{\times}$, if $z \in \operatorname{Ker}\left(\operatorname{ord}_{p}\right)$, then $z^{n_{0}} \equiv 1\left(\bmod p^{\operatorname{ord}_{p}\left(a_{0}\right)+1}\right)$.)

Now I claim that the following assertion holds:

Claim 2.2.A: For each nonnegative integer $n$ and $p \in S_{x,\langle y\rangle}$,

$$
\operatorname{ord}_{p}\left(a_{n_{0} \cdot n}\right) \leq \operatorname{ord}_{p}\left(a_{0}\right)
$$

Indeed, first observe that since $y \in \operatorname{Ker}\left(\operatorname{ord}_{p}\right)$, it follows that $y_{1}, y_{2} \in \operatorname{Ker}\left(\operatorname{ord}_{p}\right)$, which implies that $y_{1}^{n_{0}}, y_{2}^{n_{0}} \equiv 1\left(\bmod p^{\operatorname{ord}_{p}\left(a_{0}\right)+1}\right)$ (cf. the final part of the preceding paragraph). Thus, we conclude that

$$
a_{n_{0} \cdot n}-a_{0}=x_{1} \cdot\left(y_{2}^{n_{0} \cdot n}-1\right)-x_{2} \cdot\left(y_{1}^{n_{0} \cdot n}-1\right) \equiv 0\left(\bmod p^{\operatorname{ord}_{p}\left(a_{0}\right)+1}\right),
$$

i.e., $\operatorname{ord}_{p}\left(a_{0}\right)<\operatorname{ord}_{p}\left(a_{n_{0} \cdot n}-a_{0}\right)$. In particular, $\operatorname{ord}_{p}\left(a_{n_{0} \cdot n}\right) \leq \operatorname{ord}_{p}\left(a_{0}\right)$, as desired.

Next, one deduces immediately from Claim 2.2.A that $\left|a_{n_{0} \cdot n}\right| \leq\left|a_{0} \cdot x_{1} \cdot x_{2}\right|$ for sufficiently large $n$. Thus, since $|y|^{n}-|x| \leq\left|x-y^{n}\right|=\left|a_{n}\right| /\left|x_{2} \cdot y_{2}^{n}\right| \leq\left|a_{n}\right|$, and $1<|y|$, we obtain a contradiction.

Remark 2.2.1. If, in Lemma 2.2, one omits the assumption that $y \notin\{1,-1\}$, then the conclusion no longer holds. More precisely, for $x \in \mathbb{Q}^{\times}$and $y \in\{1,-1\}$, the set $S_{x,\langle y\rangle}$ is infinite if and only if $(x, y) \in\{(1,1),(1,-1),(-1,-1)\}$. Indeed, the sufficiency is immediate. To verify the necessity, observe that since $1^{2}=(-1)^{2}=1$, it follows that $x^{2} \equiv 1(\bmod p)$ for every $p \in S_{x,\langle y\rangle}$. Thus, since $S_{x,\langle y\rangle}$ is infinite, we conclude that $x^{2}=1$. In particular, since $S_{x,\langle y\rangle}=\{2\}$ when $(x, y)=(-1,1)$, the necessity follows.

Lemma 2.3. Let $x \in k^{\times}$. Then $x \in \mathbb{Q}^{\times}$if and only if $x^{\mathfrak{c}(\mathfrak{p})-1} \in 1+\mathfrak{p o}_{\mathfrak{p}}$ for all but finitely many $\mathfrak{p} \in \mathcal{V}$.

Proof. First, one verifies easily that the condition $x^{\mathfrak{c}(\mathfrak{p})-1} \in 1+\mathfrak{p o}_{\mathfrak{p}}$ implies that $x \in \operatorname{Ker}\left(\operatorname{ord}_{\mathfrak{p}}\right)$. Hence these conditions are equivalent, and moreover the image of $x \in \operatorname{Ker}\left(\operatorname{ord}_{\mathfrak{p}}\right)$ in $\operatorname{Ker}\left(\operatorname{ord}_{\mathfrak{p}}\right) /\left(1+\mathfrak{p o}_{\mathfrak{p}}\right) \stackrel{\sim}{\longrightarrow} \kappa(\mathfrak{p})^{\times}$is annihilated by $\mathfrak{c}(\mathfrak{p})-1$, i.e., contained in the prime subfield $(\simeq \mathbb{Z} / \mathfrak{c}(\mathfrak{p}) \mathbb{Z})$ of $\kappa(\mathfrak{p})$. Thus, Lemma 2.3 follows immediately from Chebotarev's density theorem. 
Proposition 2.4. Let $\phi:{ }^{\bullet} \mathcal{V} \rightarrow{ }^{\circ} \mathcal{V}$ be a map of sets.

(i) Suppose that the image of the composite ${ }^{\bullet} \mathcal{V} \stackrel{\phi}{\rightarrow}{ }^{\circ} \mathcal{V} \stackrel{\mathfrak{c}}{\rightarrow}$ Primes is of density one. Then the homomorphism of groups

$$
\operatorname{Hom}^{\phi-\mathrm{PU}}\left({ }^{\circ} k^{\times},{ }^{\bullet} k^{\times}\right) \rightarrow \operatorname{Hom}^{(\operatorname{co} \phi)-\mathrm{PU}}\left({ }^{\circ} \mathbb{Q}^{\times}, \bullet k^{\times}\right)
$$

of Lemma 1.8 is injective.

(ii) Suppose, moreover, that the image of $\bullet \mathcal{V} \stackrel{\phi}{\rightarrow}{ }^{\circ} \mathcal{V} \stackrel{\mathfrak{c}}{\rightarrow}$ Primes is cofinite. Let ${ }^{\circ} J$ be an infinite subgroup of ${ }^{\circ} \mathbb{Q}^{\times}$. Then the homomorphism of groups

$$
\operatorname{Hom}^{\phi-\mathrm{PU}}\left({ }^{\circ} k^{\times},{ }^{\bullet} k^{\times}\right) \rightarrow \operatorname{Hom}\left({ }^{\circ} J,{ }^{\bullet} k^{\times}\right)
$$

induced by the natural inclusion ${ }^{\circ} \mathrm{J} \hookrightarrow{ }^{\circ} k^{\times}$is injective.

(iii) The homomorphism of groups

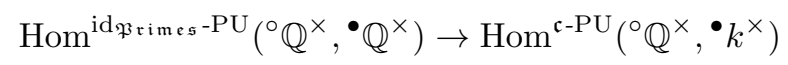

induced by the natural inclusion $\bullet^{\times} \hookrightarrow \mathbb{Q}^{\times}$is bijective.

Proof. First, we verify assertion (i). Let $\alpha:{ }^{\circ} k^{\times} \rightarrow{ }^{\bullet} k^{\times}$be a $\phi$-PU-preserving homomorphism such that $\alpha\left({ }^{\circ} \mathbb{Q}^{\times}\right)=\{1\}$. To verify that $\alpha\left({ }^{\circ} k^{\times}\right)=\{1\}$, take $x \in$ ${ }^{\circ} k^{\times}$and $\bullet \mathfrak{p} \in S[\phi ; x]$ (cf. the notation of Lemma 2.1) that is not PU-exceptional for $(\alpha, \phi)$ (cf. Definition 1.3(i)). Write ${ }^{\circ} \mathfrak{p}:=\phi(\bullet \mathfrak{p}) \in{ }^{\circ} \mathcal{V}$ and $\alpha_{\mathfrak{p}}: \kappa\left({ }^{\circ} \mathfrak{p}\right)^{\times} \rightarrow \kappa\left({ }^{\bullet} \mathfrak{p}\right)^{\times}$ for the homomorphism induced by $\alpha$ (cf. Lemma 1.5(i)). Then since $\sharp \kappa\left({ }^{\circ} \mathfrak{p}\right)=\mathfrak{c}\left({ }^{\circ} \mathfrak{p}\right)$ (cf. the definition of $S[\phi ; x]$ in Lemma 2.1), and $\alpha\left({ }^{\circ} \mathbb{Q}^{\times}\right)=\{1\}$, one verifies easily that $\alpha_{\mathfrak{p}}\left(\kappa\left({ }^{\circ} \mathfrak{p}\right)^{\times}\right)=\{1\}$, which thus implies that

$$
\alpha(x)(\bmod \bullet \mathfrak{p})=\alpha_{\mathfrak{p}}\left(x\left(\bmod ^{\circ} \mathfrak{p}\right)\right)=1 .
$$

Thus, by allowing $\mathfrak{p}$ to vary, it follows immediately from Lemma 2.1 that $\alpha(x)=1$. This completes the proof of (i).

Next, we verify assertion (ii). It follows from (i) that, to verify (ii), we may assume that ${ }^{\circ} k={ }^{\circ} \mathbb{Q}$. Let $\alpha:{ }^{\circ} k^{\times}={ }^{\circ} \mathbb{Q}^{\times} \rightarrow{ }^{\bullet} k^{\times}$be a $\phi$-PU-preserving homomorphism such that $\alpha\left({ }^{\circ} J\right)=\{1\}$. To verify that $\alpha\left({ }^{\circ} k^{\times}\right)=\{1\}$, take $x \in{ }^{\circ} k^{\times}={ }^{\circ} \mathbb{Q}^{\times}$ and $y \in{ }^{\circ} J \backslash\left({ }^{\circ} J \cap\{1,-1\}\right)$. It follows immediately from Lemma 2.2, together with our assumption that the image of $\phi:{ }^{\bullet} \mathcal{V} \rightarrow{ }^{\circ} \mathcal{V}=\mathfrak{P r i m e s}$ is cofinite, that the subset $T \subseteq \bullet \mathcal{V}$ consisting of all maximal ideals $\boldsymbol{\bullet}_{\mathfrak{p}}$ of $\boldsymbol{\bullet}_{\mathfrak{o}}$ that satisfy the following condition is infinite: If ${ }^{\circ} \mathfrak{p}:=\phi(\bullet \mathfrak{p})$, then

- $\mathfrak{p}$ is not $P U$-exceptional for $(\alpha, \phi)$,

- $x, y \in \operatorname{Ker}\left(\operatorname{ord}_{\circ}\right)$, and

- the image of $x$ in $\operatorname{Ker}\left(\operatorname{ord}_{\circ_{\mathfrak{p}}}\right) /\left(1+{ }^{\circ} \mathfrak{p} o_{\circ} \mathfrak{p}\right)$ is contained in the subgroup of $\operatorname{Ker}\left(\operatorname{ord}_{\circ} \mathfrak{p}\right) /\left(1+{ }^{\circ} \mathfrak{p} \mathfrak{o}_{\circ}\right)$ generated by the image of $y$ in $\operatorname{Ker}\left(\operatorname{ord}{ }_{\circ}\right) /\left(1+{ }^{\circ} \mathfrak{p} o_{\circ} \mathfrak{p}\right)$. 
Let $\bullet \mathfrak{p} \in T$. By the definition of $T$, there exists an integer $n$ such that $x \cdot y^{n} \in$ $1+{ }^{\circ} \mathfrak{p} o_{\mathfrak{p}}$. Thus, since we have assumed that $\alpha\left({ }^{\circ} J\right)=\{1\}$, it follows that $\alpha(x)=$ $\alpha\left(x \cdot y^{n}\right) \in 1+\mathfrak{p}^{\bullet} \mathfrak{o} \cdot \mathfrak{p}$. In particular, since $T$ is infinite, we conclude that $\alpha(x) \in$ $\bigcap_{\mathfrak{p} \in T}\left(1+\mathfrak{p}^{\bullet} \cdot \mathfrak{\bullet}_{\mathfrak{p}}\right)=\{1\}$, i.e., $\alpha(x)=1$. This completes the proof of (ii).

Finally, we verify (iii). The injectivity of the homomorphism under consideration follows immediately from the injectivity of the natural inclusion $\mathbb{Q}^{\times} \hookrightarrow$ $\bullet k^{\times}$. Next, to verify the surjectivity, take a $\mathfrak{c}-P U$-preserving homomorphism $\alpha:{ }^{\circ} \mathbb{Q}^{\times} \rightarrow{ }^{\bullet} k^{\times}$. By Lemma 2.3, $\alpha$ factors through the subgroup ${ }^{\bullet} \mathbb{Q}^{\times} \subseteq \cdot k^{\times}$ of $\bullet k^{\times}$; thus, we obtain a homomorphism ${ }^{\circ} \mathbb{Q}^{\times} \rightarrow \mathbb{Q}^{\times}$. On the other hand, since $\alpha$ is $\mathfrak{c}$ - $P U$-preserving, one verifies immediately from Lemma 1.4 that this homomorphism ${ }^{\circ} \mathbb{Q}^{\times} \rightarrow \bullet \mathbb{Q}^{\times}$is id Primes $-P U$-preserving. This completes the proof of (iii).

Remark 2.4.1. If, in Proposition 2.4(ii), one replaces our assumption that ${ }^{\circ} J$ is infinite by the assumption that ${ }^{\circ} J$ is nontrivial, then the conclusion no longer holds. Indeed, one verifies easily that the two distinct endomorphisms of $\mathbb{Q}^{\times}$obtained by mapping $x \in \mathbb{Q}^{\times}$to $x \in \mathbb{Q}^{\times}$and $x^{3} \in \mathbb{Q}^{\times}$, respectively, are contained in $\operatorname{Hom}^{\operatorname{id}_{\mathfrak{P r i m e s}}-\mathrm{PU}}\left(\mathbb{Q}^{\times}, \mathbb{Q}^{\times}\right)$and coincide on the nontrivial subgroup $\{1,-1\} \subseteq \mathbb{Q}^{\times}$.

Theorem 2.5. For $\square \in\{0, \bullet\}$, let $\square k$ be a number field; write $\square \mathcal{V}$ for the set of maximal ideals of the ring of integers of $\square k$, and $\square \mathbb{Q} \subseteq{ }^{\square}$ for the subfield of $\square k$ that is isomorphic to $\mathbb{Q}$. Let $\alpha:{ }^{\circ} k^{\times} \rightarrow{ }^{\bullet} k^{\times}$be a homomorphism of groups. Then the following conditions are equivalent:

(1) $\alpha$ arises from a homomorphism of fields ${ }^{\circ} k \rightarrow \bullet$.

(2) $\alpha$ is CPU-preserving and there exists an $x \in \mathbb{Q}^{\times} \backslash \mathbb{Z}^{\times}$such that the " $x$ " in ${ }^{\circ} k$ maps, via $\alpha$, to the " $x$ " in $\bullet$.

(3) $\alpha$ is PU-preserving and the restriction ${ }^{\circ} \mathbb{Q}^{\times} \rightarrow{ }^{\bullet} k^{\times}$of $\alpha$ to ${ }^{\circ} \mathbb{Q}^{\times} \subseteq{ }^{\circ} k^{\times}$ arises from a homomorphism of fields ${ }^{\circ} \mathbb{Q} \rightarrow \bullet^{\bullet} k$.

Proof. The implication $(1) \Rightarrow(2)$ follows immediately from Lemma 1.4, together with the various definitions involved.

Next, we verify the implication $(2) \Rightarrow(3)$. Suppose that condition $(2)$ is satisfied. First, it follows from Lemma 1.8 that we may assume that ${ }^{\circ} k={ }^{\circ} \mathbb{Q}$. Next, it follows from Proposition 2.4(iii) that we may assume that ${ }^{\circ}=\bullet \mathbb{Q}$. Since the isomorphism ${ }^{\circ} \mathbb{Q}^{\times} \stackrel{\sim}{\rightarrow} \cdot \mathbb{Q}^{\times}$determined by the identity automorphism of $\mathbb{Q}^{\times}$is contained in $\operatorname{Hom}^{\text {id }_{\mathfrak{P r i m e s}}-\mathrm{PU}}\left({ }^{\circ} \mathbb{Q}^{\times}, \bullet \mathbb{Q}^{\times}\right)$, the implication follows immediately from Proposition 2.4(ii).

Finally, we verify $(3) \Rightarrow(1)$. Suppose that (3) is satisfied. Let $\phi:{ }^{\bullet} \mathcal{V} \rightarrow{ }^{\circ} \mathcal{V}$ be such that $\alpha$ is $\phi$-PU-preserving. One verifies easily that it suffices to verify 
Claim 2.5.A: For $x, y \in{ }^{\circ} k^{\times}$, if $x+y=0$ (respectively, $x+y \neq 0$ ), then $\alpha(x)+\alpha(y)=0$ (respectively, $\alpha(x+y)=\alpha(x)+\alpha(y))$.

Since the restriction $\left.\alpha\right|_{\mathbb{Q}^{\times}}:{ }^{\circ} \mathbb{Q}^{\times} \rightarrow{ }^{\bullet} k^{\times}$arises from a homomorphism of fields ${ }^{\circ} \mathbb{Q} \rightarrow \bullet$, one verifies easily that the " -1 " in ${ }^{\circ} k^{\times}$maps, via $\alpha$, to the " -1 " in $k^{\times}$; in particular, if $x+y=0$ (i.e., $y=-x$ ), then $\alpha(x)+\alpha(y)=0$ (i.e., $\alpha(y)=-\alpha(x))$. Thus, we may assume that $x+y \neq 0$. Now, to complete the verification of Claim 2.5.A, we will prove

Claim 2.5.B: Let $\mathfrak{p} \in S[\phi ; x, y, x+y]$ (cf. the notation of Lemma 2.1) be such that $\mathfrak{p}$ is not $P U$-exceptional for $(\alpha, \phi)$. Then

$$
\alpha(x+y)\left(\bmod 1+\bullet_{\mathfrak{p}} \mathfrak{o}_{\mathfrak{p}}\right)=\alpha(x)+\alpha(y)\left(\bmod 1+\mathfrak{p}^{\bullet} \mathfrak{o} \bullet \mathfrak{p}\right) .
$$

Indeed, write ${ }^{\circ} \mathfrak{p}:=\phi\left(\bullet^{\bullet} \mathfrak{p}\right) \in{ }^{\circ} \mathcal{V}$. Since $\sharp \kappa\left({ }^{\circ} \mathfrak{p}\right)=\mathfrak{c}\left({ }^{\circ} \mathfrak{p}\right)$, there exist $x_{\mathbb{Q}}, y_{\mathbb{Q}} \in$ ${ }^{\circ} \mathbb{Q}^{\times}$such that $x_{\mathbb{Q}}, y_{\mathbb{Q}}, x_{\mathbb{Q}}+y_{\mathbb{Q}} \in \operatorname{Ker}\left(\right.$ ord $\left.\circ_{\mathfrak{p}}\right)$, and the images of $x_{\mathbb{Q}}, y_{\mathbb{Q}}$ in $\operatorname{Ker}\left(\operatorname{ord} \circ_{\mathfrak{p}}\right) /\left(1+{ }^{\circ} \mathfrak{p}^{\circ} \mathfrak{o}_{\circ} \mathfrak{p}\right)$ coincide with the images of $x, y$ in $\operatorname{Ker}\left(\operatorname{ord} \circ_{\mathfrak{p}}\right) /\left(1+{ }^{\circ} \mathfrak{p}^{\circ} \mathfrak{o}_{\circ} \mathfrak{p}\right)$, respectively. Thus, the following equalities hold:

$$
\begin{aligned}
& \alpha(x+y)\left(\bmod 1+\bullet^{\bullet} \bullet^{\mathfrak{o} \bullet \mathfrak{p}}\right)=\alpha_{\mathfrak{p}}\left(x+y\left(\bmod 1+{ }^{\circ} \mathfrak{p}^{\circ} \mathfrak{o}_{\mathfrak{p}}\right)\right) \\
& =\alpha_{\mathfrak{p}}\left(x_{\mathbb{Q}}+y_{\mathbb{Q}}\left(\bmod 1+{ }^{\circ} \mathfrak{p}^{\circ} \mathfrak{o}_{\mathfrak{p}}\right)\right) \\
& =\alpha\left(x_{\mathbb{Q}}+y_{\mathbb{Q}}\right)\left(\bmod 1+\bullet_{\mathfrak{p}} \bullet \mathfrak{o}_{\mathfrak{p}}\right) \\
& =\alpha\left(x_{\mathbb{Q}}\right)+\alpha\left(y_{\mathbb{Q}}\right)\left(\bmod 1+\mathfrak{p}^{\bullet} \mathfrak{o}_{\mathfrak{p}}\right) \\
& =\alpha_{\mathfrak{p}}\left(x_{\mathbb{Q}}\left(\bmod 1+{ }^{\circ} \mathfrak{p}^{\circ} \mathfrak{o}_{\circ \mathfrak{p}}\right)\right)+\alpha_{\mathfrak{p}}\left(y_{\mathbb{Q}}\left(\bmod 1+{ }^{\circ} \mathfrak{p}^{\circ} \mathfrak{o}_{\circ \mathfrak{p}}\right)\right) \\
& =\alpha_{\mathfrak{p}}\left(x\left(\bmod 1+{ }^{\circ} \mathfrak{p}^{\circ} \mathfrak{o}_{\circ} \mathfrak{p}\right)\right)+\alpha_{\mathfrak{p}}\left(y\left(\bmod 1+{ }^{\circ} \mathfrak{p}^{\circ} \mathfrak{o}_{\circ} \mathfrak{p}\right)\right) \\
& =\alpha(x)\left(\bmod 1+\bullet_{\mathfrak{p}} \mathfrak{o}_{\mathfrak{p}}\right)+\alpha(y)\left(\bmod 1+\mathfrak{p}_{\mathfrak{p}} \mathfrak{o}_{\mathfrak{p} \mathfrak{p}}\right) \\
& =\alpha(x)+\alpha(y)(\bmod 1+\bullet \mathfrak{p} \bullet \mathfrak{o} \cdot \mathfrak{p})
\end{aligned}
$$

—where we write $\alpha_{\mathfrak{p}}: \kappa\left({ }^{\circ} \mathfrak{p}\right)^{\times} \rightarrow \kappa(\bullet \mathfrak{p})^{\times}$for the homomorphism induced by $\alpha$ (cf. Lemma 1.5(i)); the first, third, fifth, and seventh equalities follow from the definition of $\alpha_{\mathfrak{p}}$; the second and sixth follow from the choices of $x_{\mathbb{Q}}, y_{\mathbb{Q}}$; the fourth follows from our assumption that $\left.\alpha\right|_{\circ} \mathbb{Q}^{\times}$arises from a homomorphism of fields ${ }^{\circ} \mathbb{Q} \rightarrow{ }^{\bullet} k$; the eighth follows from the various definitions involved. This completes the proof of Claim 2.5.B.

Now, by allowing $\bullet \mathfrak{p}$ to vary, it follows immediately from Claim 2.5.B, together with Lemma 2.1, that Claim 2.5.A holds. This completes the proof of $(3) \Rightarrow(1)$.

Remark 2.5.1. If, in Theorem 2.5, one replaces $\mathbb{Q}^{\times} \backslash \mathbb{Z}^{\times}$in condition (2) by either $\mathbb{Q}^{\times}$or $\mathbb{Q}^{\times} \backslash\{1\}$, then the conclusion no longer holds. Indeed, the automorphism of $\mathbb{Q}^{\times}$obtained by mapping $x \in \mathbb{Q}^{\times}$to $x^{-1} \in \mathbb{Q}^{\times}$is $C P U$-preserving, maps $-1 \in \mathbb{Q}^{\times}$ to $-1 \in \mathbb{Q}^{\times}$, but does not arise from a homomorphism of fields $\mathbb{Q} \rightarrow \mathbb{Q}$. 


\section{$\S 3$. Uchida's lemma for number fields}

In this section, we prove analogues of Uchida's lemma reviewed in the Introduction in the case of number fields (cf. Theorem 3.1 and Corollary 3.3 below).

Theorem 3.1. For $\square \in\{\circ, \bullet\}$, let ${ }^{\square} k$ be a number field; write $\square_{\mathfrak{o}} \subseteq{ }^{\square} k$ for the ring of integers of ${ }^{\square} k$, and ${ }^{\square} \mathcal{V}$ for the set of maximal ideals of $\square \mathfrak{o}$. Write Primes for the set of all prime numbers. Let $\alpha:{ }^{\circ} k^{\times} \rightarrow{ }^{\bullet} k^{\times}$be a homomorphism of groups. Then the following conditions are equivalent:

(1) $\alpha$ arises from a homomorphism of fields ${ }^{\circ} k \rightarrow{ }^{\bullet} k$.

(2) There exists a map $\phi: \bullet \mathcal{V} \rightarrow{ }^{\circ} \mathcal{V}$ over $\mathfrak{P r i m e s}$ (relative to, for each $\square \in\{\circ, \bullet\}$, the map ${ }^{\square} \mathcal{V} \rightarrow$ Primes obtained by mapping $\square \mathfrak{p} \in \square \mathcal{V}$ to the residue characteristic of $\left.{ }^{\square} \mathfrak{p}\right)$ such that, for $\bullet_{\mathfrak{p}} \in{ }^{\bullet} \mathcal{V}$, if ${ }^{\circ} \mathfrak{p}:=\phi(\bullet \mathfrak{p}) \in{ }^{\circ} \mathcal{V}$, then:

(a) For $\square \in\{\circ, \bullet\}$, if we write $\operatorname{ord}_{\square \mathfrak{p}}:^{\square} k^{\times} \rightarrow \mathbb{Z}$ for the (uniquely determined) surjective valuation of ${ }^{\square} k$ associated to $\square_{\mathfrak{p}}$, then

$$
\operatorname{ord}_{\circ}=\operatorname{ord} \bullet_{\mathfrak{p}} \circ \alpha
$$

for infinitely many $\bullet_{\mathfrak{p}} \in \bullet^{\bullet} \mathcal{V}$.

(b) For $\square \in\{\circ, \bullet\}$, if we write $\square_{\mathfrak{o}_{\mathfrak{p}}} \subseteq{ }^{\square}$ for the localization of $\square_{\mathfrak{o}}$ at the maximal ideal $\square_{\mathfrak{p}} \subseteq{ }^{\square} \mathfrak{o}$, then

$$
1+{ }^{\circ} \mathfrak{p}^{\circ} \mathfrak{o}_{\mathfrak{p}} \subseteq \alpha^{-1}\left(1+\bullet_{\mathfrak{p}}^{\bullet} \mathfrak{o} \bullet \mathfrak{p}\right)
$$

for all but finitely many $\bullet \mathfrak{p} \in \bullet \mathcal{V}$.

Proof. The implication $(1) \Rightarrow(2)$ follows immediately from Lemma 1.4, together with the well-known fact that the finite extension $\bullet k /{ }^{\circ} k$ (determined by the homomorphism of fields ${ }^{\circ} k \rightarrow{ }^{\bullet} k$ ) is unramified at all but finitely many nonarchimedean primes.

Next, we verify $(2) \Rightarrow(1)$. Suppose that condition (2) is satisfied. Now since $\alpha$ is $C P U$-preserving (cf. condition (b)), it follows from the equivalence $(1) \Leftrightarrow(2)$ of Theorem 2.5 that it suffices to verify

Claim 3.1.A: There exists an $x \in \mathbb{Q}^{\times} \backslash \mathbb{Z}^{\times}$such that the " $x$ " in ${ }^{\circ} k$ maps, via $\alpha$, to the " $x$ " in $\bullet$.

Now since $\alpha$ is $C P U$-preserving (cf. (b)), it follows immediately from Lemma 1.8, together with the well-known fact that the finite extension ${ }^{\circ} k /{ }^{\circ} \mathbb{Q}$ is unramified at all but finitely many nonarchimedean primes, that, to verify Claim 3.1.A, we may assume that ${ }^{\circ} k={ }^{\circ} \mathbb{Q}$. Next, again since $\alpha$ is $C P U$-preserving, it follows immediately from Proposition 2.4(iii) that we may assume that $\bullet k=\bullet \mathbb{Q}$. In particular, 
one verifies immediately from Remark 1.1.1 that $\phi$ is the identity automorphism of Primes.

Let $S_{(\mathrm{b})}$ be a cofinite subset of $\mathfrak{P r i m} \mathfrak{s}$ such that the displayed inclusion of (b) holds for $\bullet \mathfrak{p} \in S_{(\mathrm{b})}$, and $S_{(\mathrm{a}) \text {,(b) }}$ an infinite subset of $S_{(\mathrm{b})}$ such that the displayed equality of (a) holds for $\mathfrak{p} \in S_{(\mathrm{a}) \text {,(b) }}$. Then it follows immediately from Lemma $1.5(\mathrm{i})$ that, for each $\bullet \mathfrak{p} \in S_{(\mathrm{b})}$, there exists a uniquely determined, not necessarily positive integer $n_{\bullet}$ s such that

$$
n \bullet \mathfrak{p} \cdot \operatorname{ord}_{\mathfrak{p}}=\operatorname{ord}_{\bullet p} \circ \alpha .
$$

(Thus, if $\bullet \mathfrak{p} \in S_{(\mathrm{a}),(\mathrm{b})}$, then $n \bullet \mathfrak{p}=1$.)

For $\square \in\{\circ, \bullet\}$ and $\square_{\mathfrak{p}} \in{ }^{\square} \mathcal{V}$, write $J_{\square_{\mathfrak{p}}}(\simeq \mathbb{Z}) \subseteq{ }^{\square} k^{\times}$for the subgroup of ${ }^{\square} k^{\times}$ generated by the (element of $\square k^{\times}=\square^{\mathbb{Q}^{\times}}$corresponding to the) residue characteristic $\mathfrak{c}\left(\square_{\mathfrak{p}}\right)$ (i.e., $J_{\square_{\mathfrak{p}}}=“ \mathfrak{c}\left(\square_{\mathfrak{p}}\right)^{\mathbb{Z}}$ "). Then the various inclusions $J_{\square_{\mathfrak{p}}} \hookrightarrow{ }^{\square} k^{\times}$and the inclusion ${ }^{\square} k_{\text {tor }}^{\times} \hookrightarrow{ }^{\square} k^{\times}$(where we write ${ }^{\square} k_{\text {tor }}^{\times} \subseteq{ }^{\square} k^{\times}$for the maximal torsion subgroup of $\square k^{\times}$, i.e., $\left.\square k^{\times}="\{1,-1\} "\right)$ determine an isomorphism of abelian groups

$$
{ }^{\square} k_{\text {tor }}^{\times} \oplus\left(\bigoplus_{\square \mathfrak{p} \in \square \mathcal{V}} J_{\square \mathfrak{p}}\right) \stackrel{\sim}{\rightarrow}{ }^{\square} k^{\times} .
$$

Write $\beta:{ }^{\circ} k^{\times} \rightarrow{ }^{\bullet} k^{\times}$for the homomorphism defined as follows (cf. the above displayed isomorphism):

- $\beta$ maps $-1 \in{ }^{\circ} k^{\times}$to $-1 \in \boldsymbol{\bullet}^{\times}$.

- If $\bullet \mathfrak{p} \notin S_{(\mathrm{b})}$, then $\beta$ maps $\mathfrak{c}(\phi(\bullet \mathfrak{p})) \in{ }^{\circ} k^{\times}$to $\mathfrak{c}(\bullet \mathfrak{p}) \in{ }^{\bullet} k^{\times}$.

- If $\bullet \mathfrak{p} \in S_{(\mathrm{b})}$, then $\beta$ maps $\mathfrak{c}(\phi(\bullet \mathfrak{p})) \in{ }^{\circ} k^{\times}$to $\mathfrak{c}\left(\bullet_{\mathfrak{p}}\right)^{n \bullet \mathfrak{p}} \in \boldsymbol{\bullet}^{\times}$(concerning $n \bullet_{\mathfrak{p}}$, we refer to the final part of the preceding paragraph).

Write, moreover, $\gamma:=\alpha \cdot \beta^{-1}:{ }^{\circ} k^{\times} \rightarrow{ }^{\bullet} k^{\times}$. Then one verifies immediately from the definition of $\beta$, together with the various definitions involved, that

(i) the composite

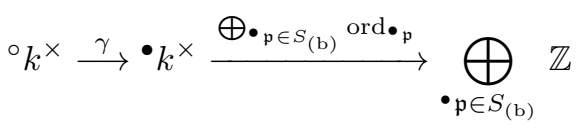

is trivial, i.e., $\gamma$ factors through the kernel $\bullet_{\text {tor }}^{\times} \oplus\left(\bigoplus_{\bullet_{\mathfrak{p} \notin S}(\mathrm{~b})} J_{\bullet_{\mathfrak{p}}}\right) \subseteq \boldsymbol{\bullet}^{\times}$of $\bigoplus \cdot p \in S_{(\mathrm{b})}$ ord $\bullet \mathfrak{p}$, and moreover

(ii) $\operatorname{Ker}(\gamma) \subseteq{ }^{\circ} k^{\times}$coincides with the subgroup of ${ }^{\circ} k^{\times}$consisting of all $x \in{ }^{\circ} k^{\times}$ such that $\alpha(x)=\beta(x)$.

Now let us observe that the kernel discussed in (i) is of finite rank, and $S_{(\mathrm{a}),(\mathrm{b})}$ is infinite. Thus, by considering the composite of the natural inclusion $\bigoplus_{\bullet \mathfrak{p} \in S_{(\mathrm{a}),(\mathrm{b})}} J_{\phi(\bullet \mathfrak{p})} \hookrightarrow{ }^{\circ} k^{\times}$and $\gamma$, we conclude from (i), (ii), together with the var- 


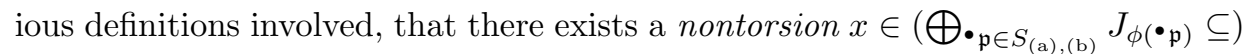
${ }^{\circ} k^{\times}$such that $\alpha(x)=x$. This completes the proof of Claim 3.1.A, hence also of Theorem 3.1.

Corollary 3.2. For $\square \in\{\circ, \bullet\}$, let ${ }^{\square} k$ be a number field. Let $\alpha:{ }^{\circ} k^{\times} \rightarrow{ }^{\bullet} k^{\times}$be a surjective group homomorphism. Then either $\alpha$ or the composite $(-)^{-1} \circ \alpha$ (i.e., the surjection ${ }^{\circ} k^{\times} \rightarrow{ }^{\bullet} k^{\times}$obtained by mapping $x \in{ }^{\circ} k^{\times}$to $\left.\alpha(x)^{-1} \in{ }^{\bullet} k^{\times}\right)$arises from an isomorphism of fields ${ }^{\circ} k \stackrel{\sim}{\rightarrow} \bullet k$ if and only if $\alpha$ is SPU-preserving.

Proof. The necessity follows from Lemma 1.4. Next, we verify the sufficiency. Suppose that $\alpha$ is $S P U$-preserving. Then from Lemma 1.5(ii), either $\alpha$ or $(-)^{-1} \circ \alpha$ satisfies condition (2) of Theorem 3.1. In particular, the sufficiency follows from Theorem 3.1.

Corollary 3.3. For $\square \in\{\circ, \bullet\}$, let $\square^{\square}$ be a number field; write $\square_{\mathfrak{o}} \subseteq{ }^{\square} k$ for the ring of integers of ${ }^{\square} k$, and ${ }^{\square} \mathcal{V}$ for the set of maximal ideals of ${ }^{\square}$ Let $\alpha$ : ${ }^{\circ} k^{\times} \rightarrow{ }^{\bullet} k^{\times}$ be a surjective group homomorphism. Then the following conditions are equivalent:

(1) $\alpha$ arises from an isomorphism of fields ${ }^{\circ} k \stackrel{\sim}{\longrightarrow} \cdot k$.

(2) There exists a map $\phi:{ }^{\bullet} \mathcal{V} \rightarrow{ }^{\circ} \mathcal{V}$ such that, for $\bullet_{\mathfrak{p}} \in{ }^{\bullet} \mathcal{V}$, if ${ }^{\circ} \mathfrak{p}:=\phi\left({ }^{\bullet} \mathfrak{p}\right) \in{ }^{\circ} \mathcal{V}$, then:

(a) For $\square \in\{\circ, \bullet\}$, if we write ord $\square_{\mathfrak{p}}$ : ${ }^{\square} k^{\times} \rightarrow \mathbb{Z}$ for the (uniquely determined) surjective valuation of $\square_{k}$ associated to $\square_{\mathfrak{p}}$, then there exist a maximal ideal $\bullet \mathfrak{p} \in \bullet \mathcal{V}$ of $\bullet \mathfrak{o}$ and a positive integer $n$ such that

$$
n \cdot \operatorname{ord}_{\circ}=\operatorname{ord}_{\bullet p} \circ \alpha .
$$

(b) For $\square \in\{\circ, \bullet\}$, if we write $\square_{\mathfrak{o} \mathfrak{p}}$ for the localization of $\square \mathfrak{o}$ at the maximal ideal $\square_{\mathfrak{p}} \subseteq \square_{\mathfrak{o}}$, then

$$
1+{ }^{\circ} \mathfrak{p}^{\circ} \mathfrak{o}_{\mathfrak{p}}=\alpha^{-1}\left(1+\mathfrak{p}^{\bullet} \mathfrak{o}_{\mathfrak{p}}\right)
$$

for all but finitely many $\bullet \mathfrak{p} \in \bullet \mathcal{V}$.

Proof. This follows immediately from Corollary 3.2, together with the various definitions involved.

Remark 3.3.1. (i) The issue of recovering the additive structure in the case of function fields has been intensively studied by M. Saïdi and A. Tamagawa (cf., e.g., $[3, \S 4]$ and $[4, \S 5]$ ). Moreover, they considered not only isomorphisms (as in Uchida's lemma - cf. Introduction) but also suitable homomorphisms between multiplicative groups. In particular, the main results of the present paper may 
also be regarded as analogues in the case of number fields of the results of SaïdiTamagawa.

(ii) One may think that the proofs of the main results of the present paper are similar to the proof of Uchida's lemma, as well as to the proofs of the results of Saïdi-Tamagawa discussed in (i) (cf., e.g., [4, Proposition 5.3]). Both the proofs consist of the following two steps:

(1) We first prove that the homomorphism under consideration between the multiplicative groups of the given global fields is compatible with the additive structures of the residue fields at various primes involved.

(2) By considering residue classes at various primes and applying the compatibility of (1), we conclude that the homomorphism under consideration between the multiplicative groups of the global fields is compatible with their additive structures.

(iii) In the case of function fields, the behaviors of minimal functions or functions with unique poles are discussed in order to perform step (1) of (ii) (cf. [3, $\S 4] ;[4, \S 5] ;[7, \S 3])$. On the other hand, in the case of number fields, to perform step (1) of (ii), the behaviors of elements of $\mathbb{Q}^{\times} \backslash \mathbb{Z}^{\times}$are discussed (cf. the proof of Theorem 2.5).

Remark 3.3.2. (i) Let us recall that Uchida's lemma (cf. Introduction), as well as the results of Saïdi-Tamagawa discussed in Remark 3.3.1(i) (cf., e.g., [4, Proposition 5.3]), were studied in the context of anabelian geometry. More precisely, in [7], Uchida's lemma was studied in order to prove that

(*) every continuous isomorphism between the absolute Galois groups of the function fields of curves over finite fields arises from an isomorphism between the original function fields.

Here, we note that an analogue of (*) for number fields was already proved (cf. [6]).

(ii) On the other hand, one may find some essential differences between the proof (given in [7]) of (*) and the proof (in [6]) of its analogue for number fields. For instance, although the proof in the case of function fields is in a "monoanabelian fashion" or "algorithmic", the proof in the case of number fields is in a "bi-anabelian fashion" or not "algorithmic" (cf., e.g., [1, Introduction and Remarks $1.9 .5,1.9 .8])$.

(iii) Now let us recall the outline of the proof of $(*)$ given in [7].

(1) First, we prove that a continuous isomorphism between the absolute Galois groups of the function fields of curves over finite fields determines a bijection between the sets of decomposition subgroups associated to primes. 
(2) Next, by means of the bijection of (1), together with class field theory, we prove that the continuous isomorphism under consideration determines an isomorphism between the multiplicative groups of the original function fields which satisfies the condition (involving ord $\square_{x}$ and $1+\mathfrak{m}_{\square}{ }_{C,}{ }_{x}$ ) in the statement of Uchida's lemma reviewed in the Introduction.

(3) Finally, by applying Uchida's lemma, we conclude that the isomorphism between the multiplicative groups of (2) determines an isomorphism between the function fields.

Here, we note that an analogue of (1) for number fields has been proved by J. Neukirch. Moreover, the main results of the present paper may be regarded as an analogue in the case of number fields of (3).

(iv) However, by the difficulty arising from the archimedean portions in the idele groups of number fields, at the time of this writing, the author is not able to prove an analogue of (2) for (arbitrary) number fields. In particular, the author is not able to obtain a proof similar to the proof in [7] of an analogue of $(*)$ for (arbitrary) number fields.

(v) On the other hand, if the number field under consideration is a subfield of an imaginary quadratic field, then one can prove immediately an analogue of (2) from the finiteness (i.e., compactness) of the group of units in the ring of integers. Thus, by means of the result of J. Neukirch, together with the main results of the present paper (cf. the final part of (iii)), one can obtain a proof similar to the proof given in [7] of an analogue of $(*)$ for such number fields. We leave the routine details to the interested reader.

\section{Acknowledgements}

The author would like to thank Kazumi Higashiyama for pointing out a minor error in the proof of Lemma 2.2 in an earlier version of the present paper. The author would like to thank the referee for comments concerning Remarks 3.3.1, 3.3 .2 .

This research was supported by Grant-in-Aid for Scientific Research (C), No. 24540016, Japan Society for the Promotion of Science.

\section{References}

[1] S. Mochizuki, Topics in absolute anabelian geometry III: Global reconstruction algorithms, RIMS Preprint 1626 (2008).

[2] P. Moree and P. Stevenhagen, A two-variable Artin conjecture, J. Number Theory 85 (2000), 291-304. Zbl 0966.11042 MR 1802718 
[3] M. Saïdi and A. Tamagawa, A prime-to- $p$ version of Grothendieck's anabelian conjecture for hyperbolic curves over finite fields of characteristic $p>0$, Publ. RIMS Kyoto Univ. 45 (2009), 135-186. Zbl 1188.14016 MR 2512780

[4] M. Saïdi and A. Tamagawa, On the Hom-form of Grothendieck's birational anabelian conjecture in positive characteristic, Algebra Number Theory 5 (2011), 131-184. Zbl 1235.14026 MR 2833788

[5] A. Tamagawa, The Grothendieck conjecture for affine curves, Compos. Math. 109 (1997), 135-194. Zbl 0899.14007 MR 1478817

[6] K. Uchida, Isomorphisms of Galois groups, J. Math. Soc. Japan 28 (1976), 617-620. Zbl 0329.12013 MR 0432593

[7] K. Uchida, Isomorphisms of Galois groups of algebraic function fields, Ann. of Math. 106 (1977), 589-598. Zbl 0372.12017 MR 0460279 